\title{
In-vitro effects of PDE5 inhibitor and statin treatment on the contractile responses of experimental MetS rabbit's cavernous smooth muscle
}

\author{
Yasin Erden ${ }^{1}$, Esat Korgalı ${ }^{1}$, Gokce Dundar ${ }^{1}$, Semih Ayan ${ }^{1}$, Gökhan Gokce ${ }^{1}$, \\ Sahin Yildirim ${ }^{2}$, Emin Yener Gultekin ${ }^{1}$ \\ ${ }^{1}$ Cumhuriyet University School of Medicine Department of Urology, Turkey; \\ ${ }^{2}$ Cumhuriyet University School of Medicine Department of Pharmachology, Turkey.
}

\begin{abstract}
Summary Objective: Hypercholesterolaemia promotes erectile dysfunction through increased superoxide formation and decreased nitric oxide bioactivity in cavernosal tissue. The role of nitric oxide on erectile function is well known. Statins have lipid lowering properties and can modulate endothelial nitric oxide bioavailability. Sildenafil, enhances smooth muscle relaxation in corpus cavernosum. We invastigated in-vitro effects of sildenafil and rosuvastatin on nonadrenergic, non-cholinergic and nitric oxide mediated cavernosal smooth musle relaxation in metabolic syndrome rabbits, since alterations in this pathway are recognised in diabetic and hypercholesterolemic erectile dysfunction.
\end{abstract}

Methods: Ten male rabbits were fed a standart diet as control group, fourty male rabbits were fed a hypercholesterolemic diet for 12 weeks. Hypercholesterolemic group were divided for without treatment, rosuvastatin treatment, sildenafil teratment, and rosuvastatin + sildenafil treatment ( $N=10$ per groups).

Results: Serum levels of cholesterol and glucose were significantly higher in the experimental group than in the control group $(p<0.05)$. After theraphy no differences were found among the groups in relaxation responses to sodium nitroprusside. The relaxation responses to carbachol and EFS were significantly reduced in metabolic syndrome group to control group $(p<0.05)$, but there were no differences between the other groups and control group. There was a signifcantly lower in-vitro relaxation response in the metabolic syndrome rabbits than in controls and the others $(p<0.05)$.

Conclusion: Both agents improve in-vitro relaxation responses of erectile tissue from metabolic syndrome rabbits to endothelial non-adrenergic, non-cholinergic and nitric oxide. This finding supports to the results of other clinical studies with these drugs.

KEY WORDS: Erectile dysfunction; Metabolic syndrome; Sildenafil; Rosuvastatin.

\section{INTRODUCTION}

Metabolic syndrome (MetS) is characterized by insulin resistance (impaired glucose tolerance, hyperinsulinemia and Type 2 Diabetes), visceral fat accumulation, dyslipidemia (low levels of high-density lipoprotein cholesterol and hypertriglyceridemia) and hypertension and increased risk of metabolic and cardiovascular diseases and erectile dysfunction (1). Subjects with MetS have a higher prevalence of sexual dysfunction, and in particular erectile dysfunction (ED) $(2,3)$. In subjects with sexual dysfunction, the prevale nce of MetS is age dependent and at midlife could affect almost the half of the population (4).

Moreover, ED has been recognized as a precursor of forthcoming CV diseases due to same factors impairing both penile and systemic vascular blood flow $(5,6)$.

Hyperglycemia, oxidative stress, and impaired lipid profiles conduce to vascular complications, including peripheral nerve perfusion deficits, which play an important role in the etiology of diabetic neuropathy. Epidemiological studies have detected that dyslipidemia is an independent risk factor for diabetic neuropathy and ED among diabetic and Mets patients (7-9).

Nowadays selective PDE5 inhibitors are used as first line therapy in the treatment of ED and are highly effective and safely. The efficacy of sildenafil was approved in vivo and in vitro, in both animal and human studies $(10,11)$. The efficacy of PDE5 inhibitors in diabetes mellitus, hyperlipidemia or dyslipidemia and hypertension extensively studied and demonstrated beneficial effect with several studies (12).

Statins are the mainstay of the management of dyslipidemia (13). Rosuvastatin is a statin, of which potency has been proved remarkably proved in reducing lowdensity lipoprotein cholesterol levels. On the other hand, rosuvastatin has extra-lipid effects and these effects are on endothelial function, oxidized low-density lipoprotein, inflammation, plaque stability, vascular remodeling, homeostasis, cardiac muscle, and components of the nervous system (14).

The effect of lipid-lowering therapies on erectile function have been studied in men with ED, along with some studies including PDE5 inhibitors (15-18). These studies sug- 
gested that as statins improve erectile function and also may ameliorate endothelial function as well, through its lipid-lowering, anti-inflammatory and antioxidant impacts (19).

The use of animal models is important in the researches of ED with dyslipidemia. A non-genomic model of MetS was developed by exposing rabbits to a high-fat diet (HFD) and dislipidemia has been shown to cause reduced erectile function in rabbits in in vitro studies, it has yet to be confirmed in a conscious in vivo model (20).

In this experimental study, we aimed to investigate the effects of sildenafil, rosuvastatin and combination of both drug therapy in normal and MetS rabbits, as the phosphodiesterase-5 (PDE-5) inhibitor sildenafil is widely used for treating erectile dysfunction (ED) and as HMGCoA (3-hydroxy-3- methylglutaryl coenzyme A) reductase inhibitor rosuvastatin is one of the most common drugs used in hyperlipidemic patients.

\section{Materials AND MEthods}

\section{Animals}

Adult male New Zealand White rabbits (Saki Yenilli Experimental Animal Production Laboratory Ankara, Turkey), weighing about $3 \mathrm{~kg}$, were individually caged under standard conditions in a temperature and humidity controlled room on a 12-hr light/dark cycle. Water and food were unrestricted during the study period. All experimental procedures were approved by animal experimental study local ethics committee of our medical school and were conducted in accordance with NIH guidelines for the care and use of laboratory animals.

\section{Creating metabolic syndrome model}

After 1 week of standard rabbit diet, animals were randomly divided into control $(n=10)$, Mets (Group a) $(\mathrm{n}=10)$ and treatment groups $(\mathrm{b}, \mathrm{c}, \mathrm{d})(\mathrm{n}=10$ each group). The control group was maintained to be fed with a standard diet (control) while Mets and the treatment groups received HFD, modified form of a previously described protocol, constituted by $0.5 \%$ cholesterol and $20 \%$ butter providing $65 \%$ of total energy need from lipids (HFD rabbit) for 12 weeks (20).

At the end of $12^{\text {th }}$ week, therapy group was divided three groups. All of therapy groups and MetS group were fed high fat diet and one group received rosuvastatin (Crestor; AstraZeneca) 20 mg/kg/day (Group b) as an oral gavage, the other group received sildenafil (Viagra; Pfizer) $5 \mathrm{mg} / \mathrm{kg} /$ day (Group c) as an oral gavage, and the remaining group received combination of rosuvastatin $20 \mathrm{mg} / \mathrm{kg} /$ day and sildenafil $5 \mathrm{mg} / \mathrm{kg} /$ day (Group d) during 6 weeks. Rabbits weights were recorded at the beginning of study, at $12^{\text {th }}$ week and at the end of the study. Blood samples for glucose, total cholesterol, triglycerides, LDL, HDL analyses were obtained from the animals via marginal ear vein at week 0 (baseline), at week 12 and at the end of the study in all groups. Plasma cholesterol, triglycerides, and glucose levels were measured by using an Automated System (ADVIA 180024004 Siemens Chemistry System; Siemens Science Medical Solution Diagnostic, NY, USA).

\section{In vitro functional studies}

All rabbits were anesthetized with isoflurane and euthanized. The entire penis was then removed, separated corpus spongiosum and urethra. Each corpus cavernosa was dissected into four strips of $5 \times 15 \mathrm{~mm}$. Isolated cavernous strips were suspended in $10 \mathrm{ml}$ organ baths containing Krebs-HCO3 solution with the following composition. (mM): $118 \mathrm{NaCl}, 4.7 \mathrm{KCl}, 2.5 \mathrm{CaCl} 2,1.2 \mathrm{MgSO} 4$, $1.2 \mathrm{KH} 2 \mathrm{PO} 4,25 \mathrm{NaHCO} 3$, and 11 glucose, $\mathrm{pH} 7.4$, at $37^{\circ} \mathrm{C}$ and bubbled with a gas mixture of $95 \%$ O 2 and $5 \%$ $\mathrm{CO} 2$. The cavernous strips were equilibrated for approximately 60 min during which the buffer solution was refreshed every $15 \mathrm{~min}$. pretension of 2 gr was applied to all strips, isometric contractions were measured with a force transducer (Grass - FT 03 Force Displacement Italy), and normalized based on strip cross-sectional area. Following the equilibration, the tissues were challenged with $124 \mathrm{mM}$ potassium chloride $(\mathrm{KCl})$ for $6 \mathrm{~min}$ and contracted all strips; washed again with fresh buffer. After more cavernous strips left to settle for the implementation of the agonist and antagonist substances, neurally evoked contractions were induced using electrical field stimulation (EFS) via platinum wire electrodes.

Isolated cavernous strips obtained from control, MetS and MetS + therapy groups were treated with $124 \mathrm{mM}$ $\mathrm{KCl}$ and responses received by KCL graphed; checked the contractility of strips before and after performing the contractile studies ( $3 \times 10-6$ tox 10-4 M). The strips were pre-contracted with $1 \times 10-5 \mathrm{M}$ fenilephrine. After the contraction reached a plateau the strips were subjected to electrical field stimulation (EFS) using ring platinum electrodes. Cavernous strips was evoked with EFS $50 \mathrm{~V}$, $0.8 \mathrm{msn} ; 2,4,8,16,32,64 \mathrm{~Hz}$ frequencies during $10 \mathrm{sec}$ after the equilibration and responses were recorded. Before applying the EFS atropine $6 x 10-5 \mathrm{M}$ and guanetidin $5 \mathrm{x} 10-5 \mathrm{M}$ was added the organ bath.

\section{Chemicals}

Carbachol, KCL, atropine, guanetidin, L- NAME, SNP, Larginine were obtained from Sigma-Aldrich.

\section{Statistical analysis}

Arithmetic averages and the standard deviations of serum values were calculated. Friedman and Wilcoxon tests were used to assess the changes in values. This experiment results were tested by Student-T test for the differences between two groups. The significance of differences between groups were evaluated with Scheffe's F test. SPSS 14.0 (SPSS, Chicago, IL, USA) was used for the statistical analysis and $\mathrm{P}<0.05$ was considered statistically significant.

\section{RESULTS}

\section{Effects of HFD to metabolic parameters in rabbits}

At the end of the first twelve weeks, there was a significant increase in plasma levels of glucose, total cholesterol, triglycerides, LDL and body weight $(\mathrm{p}<0.05)$ and plasma HDL level was decreased significantly in the HFD group. Control rabbits that were fed with a standard chow for 12 weeks did not show any significant difference in all these 
Table 1.

Analysis of serum and body weight change according to the groups.

\begin{tabular}{|c|c|c|c|c|}
\hline & Group & $\begin{array}{c}\text { Initial } \\
\text { (mg/dL } \pm \mathrm{SD})\end{array}$ & $\begin{array}{l}\text { First twelve weeks } \\
\quad(\mathrm{mg} / \mathrm{dL} \pm \mathrm{SD})\end{array}$ & $\begin{array}{l}\text { Last six weeks } \\
\text { (mg/dL } \pm \mathrm{SD} \text { ) }\end{array}$ \\
\hline Glucose & $\begin{array}{l}a \\
b \\
c \\
d\end{array}$ & $\begin{array}{l}129.54 \pm 2,30 \\
128,40 \pm 5,41 \\
127.60 \pm 2,30 \\
128.20 \pm 5,50\end{array}$ & $\begin{array}{c}* 162.42 \pm 8,67 \\
* 161,80 \pm 17,43 \\
* 159.80 \pm 8,67 \\
* 166.80 \pm 22,22\end{array}$ & $\begin{array}{c}* 178.39 \pm 10,85 \\
170,40 \pm 18,64 \\
* 184.60 \pm 10,33 \\
181.00 \pm 26,10\end{array}$ \\
\hline Total cholesterol & $\begin{array}{l}a \\
b \\
c \\
d\end{array}$ & $\begin{array}{l}46.66 \pm 2,64 \\
54,60 \pm 5,68 \\
49.80 \pm 1,64 \\
49.60 \pm 5,77\end{array}$ & $\begin{array}{c}* 85.58 \pm 3,17 \\
* 103,00 \pm 11,34 \\
* 88.80 \pm 2,17 \\
* 97.00 \pm 11,09\end{array}$ & $\begin{array}{c}* 104.62 \pm 2,98 \\
106,00 \pm 11,31 \\
* 103.80 \pm 2,77 \\
93.00 \pm 6,24\end{array}$ \\
\hline Triglyceride & $\begin{array}{l}a \\
b \\
c \\
d\end{array}$ & $\begin{array}{c}46.20 \pm 5,96 \\
43,80 \pm 73 \\
47.40 \pm 5,77 \\
47.00 \pm 7,00\end{array}$ & $\begin{array}{c}* 94.00 \pm 10,95 \\
* 114,00 \pm 17,71 \\
* 96.00 \pm 9,35 \\
* 105.60 \pm 16,08\end{array}$ & $\begin{array}{l}* 116.20 \pm 5,68 \\
113,20 \pm 20,09 \\
* 113.00 \pm 5,00 \\
110.80 \pm 14,32\end{array}$ \\
\hline$\overline{\mathrm{LDL}}$ & $\begin{array}{l}a \\
b \\
c \\
d\end{array}$ & $\begin{array}{l}12.76 \pm 1,14 \\
12,20 \pm 2,39 \\
12.60 \pm 1,14 \\
11.40 \pm 1,52\end{array}$ & $\begin{array}{l}* 23.28 \pm 3,87 \\
* 20,00 \pm 5,29 \\
* 22.20 \pm 3,63 \\
* 25.40 \pm 3,58\end{array}$ & $\begin{array}{c}* 32,54 \pm 4,92 \\
22,20 \pm 6,10 \\
* 28.60 \pm 4,22 \\
26.00 \pm 3,81\end{array}$ \\
\hline$\overline{H D L}$ & $\begin{array}{l}a \\
b \\
c \\
d\end{array}$ & $\begin{array}{l}20,51 \pm 4,96 \\
21,60 \pm 4,83 \\
21.00 \pm 4,36 \\
19.80 \pm 5,63\end{array}$ & $\begin{array}{l}* 12,47 \pm 3,85 \\
* 11,00 \pm 2,92 \\
* 11.60 \pm 2,07 \\
* 12.00 \pm 1,22\end{array}$ & $\begin{array}{c}* 10.65 \pm 2,69 \\
10,40 \pm 3,29 \\
* 10.20 \pm 2,17 \\
12.60 \pm 1,67\end{array}$ \\
\hline Body weight & $\begin{array}{l}a \\
b \\
c \\
d\end{array}$ & $\begin{array}{c}3254,55 \pm 82,36 \\
3220.80 \pm 129,22 \\
3183.80 \pm 73,96 \\
3147.40 \pm 98,60\end{array}$ & $\begin{array}{l}* 3396,65 \pm 122,32 \\
* 3373.40 \pm 114,80 \\
* 3357.80 \pm 114,28 \\
* 3414.20 \pm 143,55\end{array}$ & $\begin{array}{c}* 3566,85 \pm 107,67 \\
3418.40 \pm 250,58 \\
* 3492.80 \pm 104,84 \\
3492.20 \pm 155,72\end{array}$ \\
\hline
\end{tabular}

variables. At the end of the treatment phase which is the second phase of the study, plasma levels of glucose, total cholesterol, triglycerides, LDL, HDL in group $\mathrm{b}$ and $\mathrm{d}$ were comparable with in the initial levels of the study and with the levels in control group. Significant changes in plasma levels of glucose, total cholesterol, triglycerides, LDL, HDL observed in the first twelve weeks were observed to continue in group a and c (Table 1).

In-vitro contraction and relaxation responses

There was no difference in contractile response to $\mathrm{KCl}$ (124mM) between corporal strips from the all of MetS

Figure 1.

Concentration-response curve for phenylephrine.

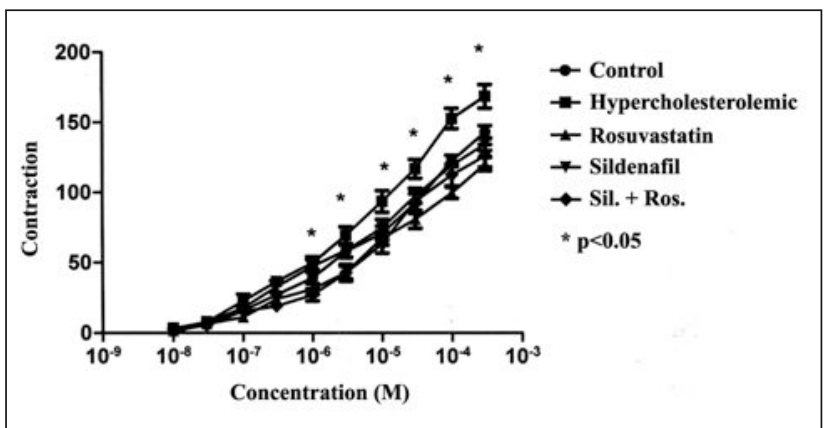

groups and control group. But there was a significant increase in contractile response to fenilefrin in corporal strips MetS as compared to control group and other MetS groups which received any of the therapeutic intervention (Figure 1).

Relaxation of corpus cavernosum smooth muscle in response to carbachol was significantly decreased in strips from the MetS group compared to controls and MetS + therapy groups (Figure 2).

When the strips incubated with 3 x 10-5 M L-NAME, the relaxation response to carbachol was inhibated and basal tonus was increased.

Figure 2.

Relaxation responses for carbachol.

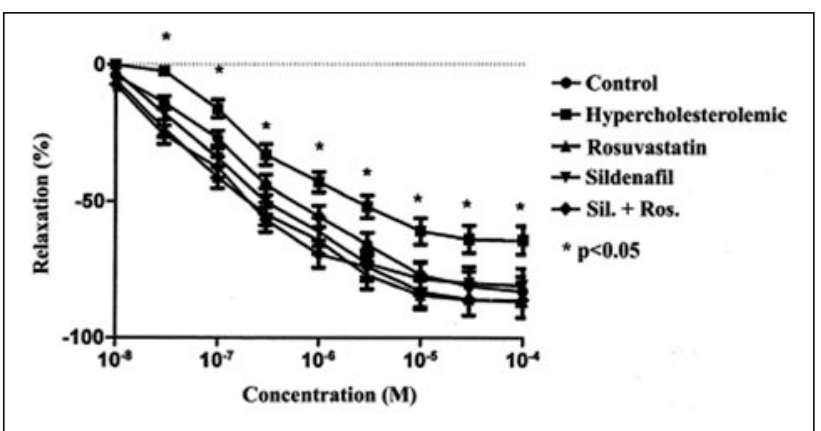


Figure 3.

Relaxation responses for sodium nitroprusside.

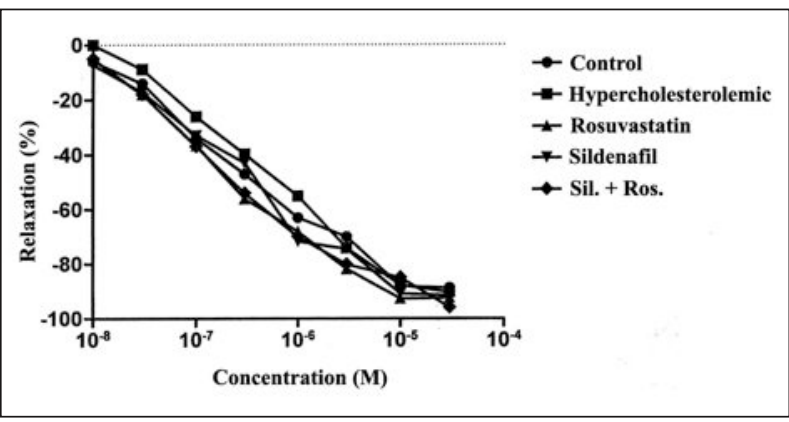

Figure 4.

Relaxation responses for EFS.

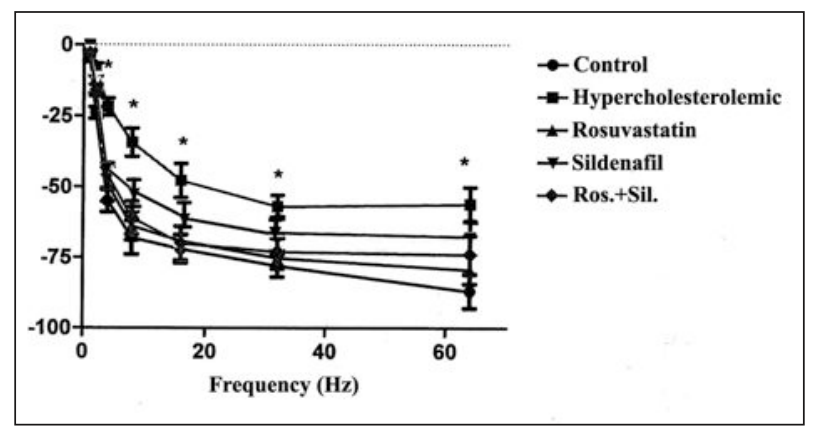

There was not significant difference in relaxation responses to sodium nitroprusside in all groups compared control group (Figure 3 ).

EFS following phenylephrine precontraction in the presence of 1 x 10-5 M/l atropine and 4x10-5 M/l guanethidine produced frequency-dependent NANC relaxation. Relaxtion responses to EFS was decreased when strips incubated with $3 \times 10-5 \mathrm{M}$ L-NAME and relaxation response to EFS return with $4 \times 10-5 \mathrm{M}$ L-arginin. This findig supported the previous detection.

Relaxation of corpus cavernosum smooth muscle in response to EFS was significantly less in strips from the MetS group than controls and all MetS + theraphy groups at all frequencies. There was no significant difference between theraphy groups each other and control group (Figure 4).

\section{Discussion}

In this study, we aimed to investigate in vitro effects of systemic treatments of rosuvastatin, sildenafil and combination of these two agents on contractile responses of cavernous tissue in experimental metabolic syndrome model. The corporal tissue strips of MetS rabbits were observed to have significantly decreased in vitro erectile responses than that of the control group whereas in MetS groups received a treatment either with a PDE5 inhibitor or with a statin, in vitro erectile responses were comparable to each other and to control goup.

Epidemiological studies have demonstrated that elevated serum cholesterol and diminished high-density lipopro- tein (HDL) cholesterol levels are associated with an increased risk of erectile dysfunction (ED). Whether restoring a dyslipidemic profile will result in a reduced risk of developing ED has not been established. Similarly, it is not known if such an intervention will improve symptoms in patients with established ED. The situation is even more complex by the likelihood that one of the rarer side effects of statins is ED (21).

Penile erection is the result of relaxation of smooth muscles in the cavernous sinusoids and associated blood vessels (23). Smooth muscle relaxation is mediated primarily by nitric oxide (NO), which one of the most potent endogenous smooth muscle relaxants. $\mathrm{NO}$ is synthesized by neuronal NO synthase (nNOS) in the autonomic postganglionic parasympathetic nerves (nitrergic nerves) 2, 3 and by endothelial NOS in the endothelium lining the blood vessels and cavernous sinusoids $(23,24)$.

The negative effects of hyperlipidemia on erectile function are revealed by clinical and experimental studies. Azadzoi et al. (25) showed in cavernous tissues of rabbits that atherosclerosis due to hyperlipidemia decreases the activity of NOS, upregulates the production of thromboxane and prostaglandin, and accordingly this negatively affects smooth muscle relaxation occurring in response to electrical stimulation which forms the basis of erection. Kim et al. (26) also found that the negative effects of hyperlipidemia on cavernous smooth muscle relaxation are related to the contractile effect of oxidized low-density lipoprotein, release of superoxide radicals and elevated levels of NOS inhibitors.

In our study, in accordance with the findings of these studies, we found that relaxation responses in untreated hypercholesterolemia were significantly reduced. Similarly, Firoozi et al. reported in their study that relaxation responses were significantly decreased in the hypercholesterolemic group. In their study, when sildenafil and vardenafil were added in vitro to hypercholesterolemic group relaxation response to EFS significantly increased however failed to reach the level of response of the control group (20). In our study, apart from the situation indicated above, in all groups where MetS model was formed and systemic therapy was added, we found that when atropine and guanetidin added to the therapy, the relaxation response to EFS was significantly increased compared to untreated MetS group. Moreover, these responses were similar compared to responses of the control group.

SNP-induced relaxation responses were similar in all groups and this suggested that the problem existed until the NO release process. This finding indicates that systemic rosuvastatin, sildenafil and combination therapy prevent the development of endothelial dysfunction and ED in hypercholesterolemic group. The beneficial effects of daily use of PDE5 inhibitors on erectile function have been shown in many experimental and clinical studies. It is shown that chronic PDE5 inhibition improves endothelial function, protects vascular smooth muscle and reduces fibrotic changes in diabetic patients with cardiovascular disease $(27,28)$. Chen et al. (29) reported antioxidative effect of the chronic use of tadalafil prevented the development of diabetic ED with diabetic rats.

A study investigating the effect of chronic use of sidenafil 
in a different ED model formed with cavernous nerve injury in rats showed that sildenafil, independent from endogenous iNOS, prevents histological changes that occur because of cavernous nerve damage (30).

In our study, we have demonstrated that the daily use of sildenafil in hypercholesterolemic rabbits of our MetS model significantly improved cavernosal tissue relaxation responses.

Statins are the most commonly used group of drugs in the treatment of dyslipidemia. Recently it has been reported that beside lipid-lowering effects, the beneficial effects of these drugs on vascular system process through modulating eNOS, iNOS, nNOS enzymes, causing relaxation of smooth muscle cells, affecting the anti-inflammatory and antioxidant mechanisms (14).

Nangle et al. (31) investigated the effect of use of rosuvastatin on nitric oxide-dependent function in aorta and corpus cavernosum of diabetic mice and reported that rosuvastatin, independent from its extra-lipid effects, restores the defective NO-mediated nerve and vascular function in diabetic mice. Moreover, dependent on its cholesterol biosynthesis pathway inhibition and anti inflammatory effects, rosuvastatin restores relaxation response which is reduced due to diabetes. Maximum NO-dependent nonadrenergic, noncholinergic nervemediated relaxations of cavernosum were reduced 25 $33 \%$ by diabetes. Rosuvastatin treatment prevented $75 \%$ and reversed $71 \%$ of this diabetic deficit.

Morelli et al. (32) reported that atorvastatin did not act on glycemia, plasma lipid levels or the hypogonadal condition in experimental diabetic rat models. In diabetic rabbits, atorvastatin improved the erectile response to electrical stimulation of the cavernous nerve and normalized the sildenafil effect on erectile function which is strongly decreased in diabetic patients. In accordance with literature, results of our study demonstrated that rosuvastatin therapy that was administered to rabbits of MetS model provided significantly increased relaxation responses of cavernosal strips.

In the literature, although there are experimental studies available concerning various vascular pathologies in which effects of use of systemic statins in combination with PDE5 inhibitors were evaluated $(33,34)$. Our study is the first in vitro study investigating the effect of use of systemic rosuvastatin in combination with sildenafil on cavernosal tissue in MetS model. We observed that the treatment with either sildenafil or rosuvastatin significantly improves cavernosal smooth muscle relaxation responses in created MetS model rabbits as compared to the untreated MetS group. However, the combination of these two drugs does not yield an additional advantage in terms of cavernosal tissue contraction responses. The lack of an additive restoring effect of combined treatment might be attributed to NO dependent effect which was alraedy provided by either of two drugs.

\section{RefEREnCES}

1. Després JP, Lemieux I. Abdominal obesity and metabolic syndrome. Nature 2006; 14:881-7.

2. Esposito K, Giugliano D. Obesity, the metabolic syndrome, and sexual dysfunction. Int J Impot Res 2005; 17:391-8.
3. Esposito K, Giugliano F, Martedi E, et al. High proportions of erectile dysfunction in men with the metabolic syndrome. Diabetes Care 2005; 28:1201-3.

4. Corona G, Mannucci E, Forti G. Hypogonadism, ED, metabolic syndrome and obesity: A pathological link supporting cardiovascular diseases. Int J Androl 2009; 32:587-98.

5. Corona G, Forti G, Maggi M. Why can patients with erectile dysfunction be considered lucky? The association with testosterone defciency and metabolic syndrome. Aging Male 2008; 11:193-9.

6. Inman BA, Sauver JL, Jacobson DJ, et al. A population-based, longitudinal study of erectile dysfunction and future coronary artery disease. Mayo Clin Proc 2009; 84:108-13.

7. Cameron NE, Eaton SEM, Cotter MA, et al. Vascular factors and metabolic interactions in the pathogenesis of diabetic neuropathy. Diabetologia 2001; 44:1973-1988

8. Sima AAF, Sugimoto K. Experimental diabetic neuropathy: an update Diabetologia 1999; 42:773-788.

9. Tesfaye S, Stevens LK, Stephenson JM, et al. Prevalence of diabetic peripheral neuropathy and its relation to glycaemic control and potential risk factors: the EURODIAB IDDM Complications Study. Diabetologia 1996; 39:1377-1384.

10. Goldstein I, Lue TF, Padma-Nathan H, et al. Oral sildenafil in the treatment of erectile dysfunction. The Sildenafil Study Group. N Engl J Med 1998; 338:1397-1404.

11. Choi S, O'Connell L, Min K, et al. Efficacy of vardenafil and sildenafil in facilitating penile erection in an animal model. J Androl 2002; 23:332-7.

12. Nehra A. Erectile dysfunction and cardiovascular disease: efficacy and safety of phosphodiesterase type 5 inhibitors in men with both conditions. Mayo Clin Proc. 2009; 84:139-48.

13. Ong HT. The statin studies from targeting hypercholesterolaemia to targeting the high-risk patient. QJM. 2005; 98:599-614.

14. Kostapanos MS, Milionis HJ, Elisaf MS. An overview of the extra-lipid effects of rosuvastatin. J Cardiovasc Pharmacol Ther. 2008; 13:157-74.

15. Bruckert E, Giral P, Heshmati HM, et al. Men treated with hypolipidaemic drugs complain more frequently of erectile dysfunction. J Clin Pharm Ther 1996; 21:89-94.

16. Saltzman EA, Guay AT, Jacobson J. Improvement in erectile function in men with organic erectile dysfunction by correction of elevated cholesterol levels: A clinical observation. J Urol 2004; 172:255-8.

17. Bank AJ, Kelly AS, Kaiser DR, et al. The effects of quinapril and atorvastatin on the responsiveness to sildenafil in men with erectile dysfunction. Vasc Med 2006; 11:251-7.

18. Solomon H, Samarasinghe YP, Feher MD, et al. Erectile dysfunction and statin treatment in high cardiovascular risk patients. Int J Clin Pract 2006; 60:141-5.

19. Miner M, Billups KL. Erectile dysfunction and dyslipidemia: relevance and role of phosphodiesterase type-5 inhibitors and statins. J Sex Med. 2008; 5:1066-78.

20. Firoozi F, Longhurst PA, White MD. In vivo and in vitro response of corpus cavernosum to phosphodiesterase-5 inhibition in the hypercholesterolaemic rabbit. BJU international. 2005; 96:164-8.

21. Schachter M. Erectile dysfunction and lipid disorders. Curr Med Res Opin. 2000; 16 (suppl-1):s9-s12. 
22. Gratzke C, Angulo J, Chitaley K, et al. Anatomy, physiology, and pathophysiology of erectile dysfunction. J Sex Med 2010; 7:445-475.

23. Moncada S, Higgs A, Furchgott R. International Union of Pharmacology Nomenclature in Nitric Oxide Research. Pharmacol Rev 1997; 49:137-142.

24. Cellek S. Let's make NO mistake! Int J Impot Res 2005; 17:388-389.

25. Azadzoi KM, Kim N, Brown M, et al. Endothelium-derived nitric oxide and cyclooxygenase products modulate corpus cavernosum smooth muscle tone. J Urol. 1992; 147:220-225.

26. Kim SC, Kim IK, Seo KK, et al. Involvement of superoxide radical in the impaired endothelium-dependent relaxation of cavernous smooth muscle in hypercholesterolemic rabbits. Urol Res. 1997; 25:341-346.

27. Francis SH, Corbin JD. PDE5 Inhibitors: Targeting erectile dysfunction in diabetics. Opin Pharmacol. 2011; 11:683-8.

28. Deyoung L, Chung E, Kovac JR, et al. Daily use of sildenafil improves endothelial function in men with type 2 diabetes. J Androl. 2012; 33:176-80

29. Chen Y, Li XX, Lin HC, et al. The effects of long-term administration of tadalafil on STZ-induced diabetic rats with erectile dys- function via a local antioxidative mechanism. Asian J Androl. 2012; 14:616-20.

30. Kovanecz I, Rambhatla A, Ferrini M, et al. Long-term continuous sildenafil treatment ameliorates corporal veno-occlusive dysfunction (CVOD) induced by cavernous nerve resection in rats. Int J Impot Res. 2008; 20:202-12.

31. Nangle MR, Cotter MA, Cameron NE. Effects of rosuvastatin on nitric oxide-dependent function in aorta and corpus cavernosum of diabetic mice: relationship to cholesterol biosynthesis pathway inhibition and lipid lowering. Diabetes. 2003; 52:2396-402.

32. Morelli A, Chavalmane AK, Filippi, et al. Atorvastatin ameliorates sildenafil-induced penile erections in experimental diabetes by inhibiting diabetes-induced RhoA/Rho- kinase signaling hyperactivation. J Sex Med. 2009; 6:91-106.

33. Zhang WH, Liu CP, Zhang YJ, et al. Additive effect of tadalafil and simvastatin on monocrotaline-induced pulmonary hypertension rats. Scand Cardiovasc J. 2012; 46:374-80.

34. Wang QM, Wei Y, Zheng Y, et al. Efficacy of combined atorvastatin and sildenafil in promoting recovery after ischemic stroke in mice. Am J Phys Med Rehabil. 2013; 92:143-50.

\section{Correspondence}

Yasin Erden, MD

Esat Korgah, MD (Corresponding Author)

estkorgali@hotmail.com, estkorgali@gmail.com

Gokce Dundar, MD

Semih Ayan, MD

Gökhan Gokce, MD

Emin Yener Gultekin, MD

Cumhuriyet University School of Medicine - Department of Urology

Kampus, Merkez - Sivas (Turkey) 58140

Sahin Yildirim, MD

Cumhuriyet University School of Medicine Department of Pharmachology 